\title{
Non-Invasive Detection of Adulterated Olive Oil in Full Bottles Using Time-Domain NMR Relaxometry
}

\author{
Poliana M. Santos, ${ }^{a}$ Flávio Vinicius C. Kock, ${ }^{b}$ Maiara S. Santos, ${ }^{b}$ Carlos Manuel S. \\ Lobo, ${ }^{b}$ André S. Carvalho ${ }^{b}$ and Luiz Alberto Colnago ${ }^{*, c}$
}

\author{
${ }^{a}$ Departamento Acadêmico de Química e Biologia, Universidade Tecnológica Federal do Paraná, \\ Rua Deputado Heitor de Alencar, 5000, 81280-340 Curitiba-PR, Brazil \\ ${ }^{b}$ Instituto de Química de São Carlos, Universidade de São Paulo, Avenida Trabalhador \\ São Carlense, 400, 13566-590 São Carlos-SP, Brazil
}

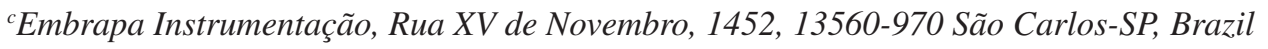

\begin{abstract}
A fast procedure using time-domain nuclear magnetic resonance (TD-NMR) to detect olive oil adulteration with polyunsaturated vegetable oils in filled bottles is proposed. The ${ }^{1} \mathrm{H}$ transverse relaxation times $\left(\mathrm{T}_{2}\right)$ of 37 commercial samples were measured using low-field nuclear magnetic resonance (LF-NMR) spectrometer and a unilateral nuclear magnetic resonance (UNMR) sensor. Results obtained with LF-NMR revealed better feasibility when compared with the UNMR sensor, with higher signal-to-noise $(\mathrm{S} / \mathrm{N})$ ratio and larger difference in the $\mathrm{T}_{2}$ decays. Principal component analysis (PCA) exhibited tight and well-separated clusters of pure olive oil (OO), pure soybean oil ( $\mathrm{SO}$ ), and blends of OO/SO (adulterated samples). Soft independent modeling of class analogies analysis (SIMCA) classification model indicated that five brands of olive oil commercialized in Brazil were adulterated with polyunsaturated fatty acids, further confirmed by high-resolution NMR. Overall, LF-NMR provided a fast procedure for screening olive oil authenticity directly in the sealed bottles.
\end{abstract}

Keywords: time-domain NMR, low-field NMR, adulteration, olive oil

\section{Introduction}

Olive oil stands out as the most expensive edible oil and is among the most frequently adulterated food products. ${ }^{1,2}$ Olive oil is often adulterated with cheaper vegetable oils, including soybean, corn, sunflower, cotton, hazelnut, peanut, palm and many others. ${ }^{1-4}$ Such practice is unfair not only to consumers, but also to the honest farmers and olive oil industries. Therefore, the detection of olive oil adulteration has driven the development of rapid, reliable, non-destructive, non-invasive and cost effective analytical procedures that could be applied at any stage of the distribution chain.

Currently, almost all analytical instrumental methods are used to detect olive oil adulterations. Gas and liquid chromatography either coupled or not with mass spectrometry have been the standard analytical methods. 5,6 However, these methods are laborious (extensive sample

*e-mail: luiz.colnago@embrapa.br preparation/derivatization), time consuming, and quite expensive. Consequently, several alternative methods have been proposed, including vibrational spectroscopies (UV-Vis, near infrared (NIR), middle infrared (MIR) and Raman), electrochemical and high- and mid-resolution ${ }^{1} \mathrm{H}$, ${ }^{13} \mathrm{C}$ and ${ }^{31} \mathrm{P}$ nuclear magnetic resonance (NMR) methods. ${ }^{7-10}$ Although these methods have become an attractive alternative due to the ability to analyze samples with little or no sample preparation, they do not allow the analysis in a completely non-invasive fashion. Recently, the feasibility of time-domain nuclear magnetic resonance (TD-NMR) to detect olive oil adulteration in a non-invasive way was explored by $\mathrm{Xu}$ et al. ${ }^{4}$ In their study, a low cost unilateral NMR sensor (magnet and surface coil) was applied to detect olive oil adulteration with sunflower or red palm oil. The procedure based on a two-dimensional (2D) pulse sequence that separates the ${ }^{1} \mathrm{H}$ transverse relaxation time $\left(\mathrm{T}_{2}\right)$ and self-diffusion coefficients (D). Although the procedure allowed the analysis in a sealed bottle, it could take several hours due to the need of acquiring a large number of NMR 
signals using different echo times to encode the diffusion effect on the $\mathrm{T}_{2}$ decay.

In this paper, the advantages and limitations of rapid, non-invasive TD-NMR procedure to detect olive oil adulteration with polyunsaturated vegetable oils in intact original bottle were investigated. The performance of low-field nuclear magnetic resonance (LF-NMR) spectrometer and unilateral nuclear magnetic resonance (UNMR) sensor were compared in order to obtain a procedure for field applications, i.e., analyses by dealer and consumer in distribution centers and grocery stores.

\section{Experimental}

\section{Samples}

Corn, canola, sunflower, soybean, hazelnut and olive oil samples were used in this study. All samples were acquired at local stores in the city of São Carlos, SP, Brazil, totaling 37 commercial oil brands from eight different countries (Brazil, Chile, Italy, Spain, Portugal, Greece and Argentina). The samples were stored and analyzed at $23.0 \pm 0.5^{\circ} \mathrm{C}$.

The fatty acid contents were determined by gas chromatography (GC) in a system (Shimadzu GC-14B model) equipped with flame ionization detector (FID). The GC separation was evaluated in the omega wax 250 capillary column. The analysis method required the conversion of the triglycerides contained in the olive and soybean oil samples into their corresponding fatty acid methyl esters (FAME) through transesterification. ${ }^{11}$

\section{NMR analysis}

\section{TD-NMR analysis in the LF-NMR spectrometer}

The ${ }^{1} \mathrm{H}$ transverse relaxation time $\left(\mathrm{T}_{2}\right)$ measurements were performed in a 0.23 Tesla SLK-IF-1399 NMR spectrometer (Spinlock Magnetic Resonance Solution, Cordoba, Argentina). The LF-NMR spectrometer was equipped with a permanent magnet Halbach array with $10 \mathrm{~cm}$ bore and probe with solenoid coil. The spectrometer was connected to the Apollo console (Tecmag, Houston, TX, USA). The $\mathrm{T}_{2}$ relaxation curves were evaluated using Carr-Purcell-Meiboom-Gill (CPMG) pulse sequence with $\pi / 2$ pulse width of $35 \mu \mathrm{s}$, time between echoes of $0.5 \mathrm{~ms}$, 1000 echoes, and a recycle delay of $1 \mathrm{~s}$. Four scans were accumulated to increase the signal-to-noise $(\mathrm{S} / \mathrm{N})$ ratio, with total measurement time in the order of $1 \mathrm{~s}$.

\section{TD-NMR analysis in the UNMR sensor}

The homemade UNMR sensor, operating at $24.3 \mathrm{MHz}$ for ${ }^{1} \mathrm{H}$ nucleus, was used to collect the $\mathrm{T}_{2}$ relaxation curves. The UNMR sensor was assembled using four axially magnetized NdFeB alloy blocks $(2.54 \times 2.54 \times 1.27 \mathrm{~cm})$ in a classical steel yoke and connected to an Apollo console (Tecmag, Houston, TX, USA). ${ }^{12}$ The $\mathrm{T}_{2}$ relaxation curves were evaluated using a CPMG pulse sequence with $\pi / 2$ pulse width of $3 \mu$ s, time between echoes of $0.1 \mathrm{~ms}$, 1000 echoes, and a recycle delay of $1 \mathrm{~s}$. Five hundred scans were accumulated in order to increase the $\mathrm{S} / \mathrm{N}$ ratio, with total measurement time in the order of $5 \mathrm{~s}$.

\section{${ }^{1} \mathrm{H}$ high-resolution NMR analysis}

${ }^{1} \mathrm{H}$ high-resolution NMR ( ${ }^{1} \mathrm{H}$ NMR) analyses were performed in a 14.1 Tesla Ascend 600 NMR spectrometer (Bruker, Germany) equipped with a $5 \mathrm{~mm}$ probe. The spectra were acquired using $30^{\circ}$ pulses, acquisition time of $4.63 \mathrm{~s}$ (32k points), recycle delay of $4 \mathrm{~s}$, and accumulation of 4 transients. Before the analyses, the samples were dissolved in $\mathrm{CDCl}_{3}$.

\section{Data analysis}

The $\mathrm{T}_{2}$ relaxation curves collected in the LF-NMR spectrometer and in the UNMR sensor were normalized (as to the maximum amplitude of the first echo) and analyzed by exponential fitting, inverse Laplace transform (ILT), principal component analysis (PCA), and soft independent modeling of class analogies (SIMCA).

The exponential fitting of the $\mathrm{T}_{2}$ relaxation curves was performed by mono- and bi-exponential functions available in Origin ${ }^{\circledR}$ software, version 9.1 (OriginLab, Northampton, MA, USA). The ILT was performed using the method described by Borgia et al..$^{13}$

PCA was used to find the main variations in the full $\mathrm{T}_{2}$ relaxation curve decays, whereas SIMCA was used to develop classification models based on oil type. PCA and SIMCA models were performed in Pirouette ${ }^{\circledast}$ software, version 4.5 (Infometrix Inc., Woodville, WA, USA). Before the analyses, the $\mathrm{T}_{2}$ relaxation curves were mean-centered.

The ${ }^{1} \mathrm{H}$ spectra obtained in the high-resolution NMR spectrometer were analyzed using topspin software, version 3.2 (Bruker, Germany). The areas of the signals at 2.3 (attributed to oleic acid) and from 5.5 to $5.9 \mathrm{ppm}$ (assigned to linoleic acid) were calculated and the ratio was determined and used to investigate the presence of polyunsaturated fatty acids in olive oil and other oil samples. ${ }^{3}$ Ratios lower than 0.2 indicated low contents of polyunsaturated fatty acids, whereas ratios greater than 0.2 indicated high contents of polyunsaturated fatty acids, normally as a result of the presence of soybean, sunflower, corn, canola and other oils. 


\section{Results and Discussion}

\section{Comparison of the performances of UNMR sensor and LF-NMR spectrometer}

Figure 1 shows the $\mathrm{T}_{2}$ relaxation curves of pure olive oil (OO) and soybean oil (SO) samples obtained by CPMG pulse sequence using the UNMR sensor (Figure 1a) and the LF-NMR spectrometer (Figure 1b). Although five hundred relaxation curves were average in UNMR signals the $\mathrm{S} / \mathrm{N}$ ratios were approximately ten-fold lower than those obtained with LF-NMR spectrometer. This large reduction in the $\mathrm{S} / \mathrm{N}$ ratio in the $\mathrm{T}_{2}$ relaxation times measured in the UNMR sensor could be associated with the strong static magnetic field gradient, $10 \mathrm{~T} \mathrm{~m}^{-1}$, which restricted the analysis to a very thin slice (approximately $0.1 \mathrm{~mm}$ ) of the sample at $5 \mathrm{~mm}$ from the sensor surface. Conversely, the relaxation curves in the LF-NMR spectrometer provided higher $\mathrm{S} / \mathrm{N}$ ratio and much shorter (five-fold) measuring time due to the good magnetic field homogeneity over a large portion of the bore volume. ${ }^{14}$ In the Halbach array, the magnetic field was perpendicular to the cylinder axis, allowing the use of sensitive solenoid coils to excite and detect NMR signals.

Moreover, no differences in the $\mathrm{T}_{2}$ values were observed between $\mathrm{OO}$ and SO samples analyzed in the UNMR sensor. In this sensor, the $\mathrm{T}_{2}$ relaxation time was also dependent on the self-diffusion coefficient (D) due to the high inhomogeneity of the magnet. $\mathrm{T}_{2}$ and $\mathrm{D}$ had opposite effects on the decay, reducing the difference between the two oils. On the other hand, the analysis performed in the LF-NMR showed a visible difference in the $T_{2}$ relaxation times of the two oils (Figure 1b).

For field applications, safety requirements denote an additional problem. The strong magnetic fields used in

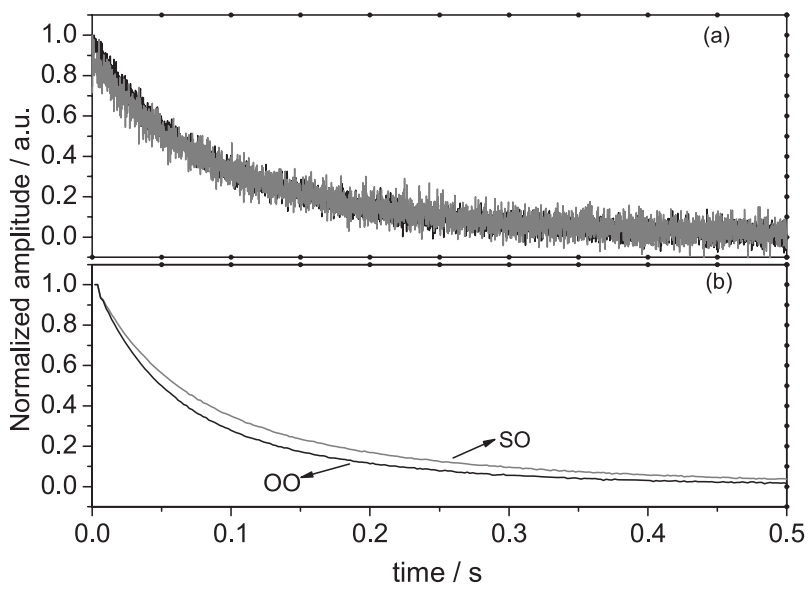

Figure 1. $\mathrm{T}_{2}$ relaxation curves of olive oils $(\mathrm{OO})$ (black line) and soybean oils (SO) (gray line) samples obtained by a CPMG pulse sequence using the (a) UNMR sensor; (b) LF-NMR spectrometer.
NMR spectrometers might attract metallic pieces and lead to serious accidents. In this sense, the limitations and advantages of the UNMR sensor and the LF-NMR spectrometer for field application were investigated. Results showed that the UNMR sensor, at distance of $1 \mathrm{~cm}$ from the surface, attracted a medium size plier ( $300 \mathrm{~g}$ ) by the tip, imposing a force of approximately $100 \mathrm{~N}$. This is equivalent to an increase of more than twenty five times the plier weight. Conversely, the attracting force of the permanent magnet Halbach array is minimal $(1 \mathrm{~N}$ at $1 \mathrm{~cm}$ from the entrance of the magnet bore). These results indicate that only professional personnel should use the UNMR sensor, whereas the Halbach magnet could be used in field applications without any risks for the analyst.

Given these results, the development of a non-invasive screening method for the detection of adulterated olive oil was evaluated using only the LF-NMR spectrometer.

\section{Analysis of oil samples using the LF-NMR spectrometer}

Figure 2 shows the normalized $\mathrm{T}_{2}$ relaxation decays of OO, SO, and OO/SO blends (adulterated samples). Table 1 shows the fatty acid composition of $\mathrm{OO}$ and SO as determined by gas chromatography. The OO/SO blends were prepared by the addition of SO into OO in order to obtain samples with 12.5, 25.0, 50.0 and $75.0 \%$ of adulteration. OO sample showed the fastest decay (shortest $\mathrm{T}_{2}$ ), whereas $\mathrm{SO}$ sample led to the longest decay (longest $\mathrm{T}_{2}$ ), indicating a short $\mathrm{T}_{2}$ relaxation with increasing $\mathrm{OO}$ concentrations. This variation in $\mathrm{T}_{2}$ values could be associated with the differences in oil viscosity, which in turn is dependent on the major fatty acids present in the oil. The composition of $\mathrm{OO}$ and $\mathrm{SO}$ differed significantly in terms of unsaturated fatty acids

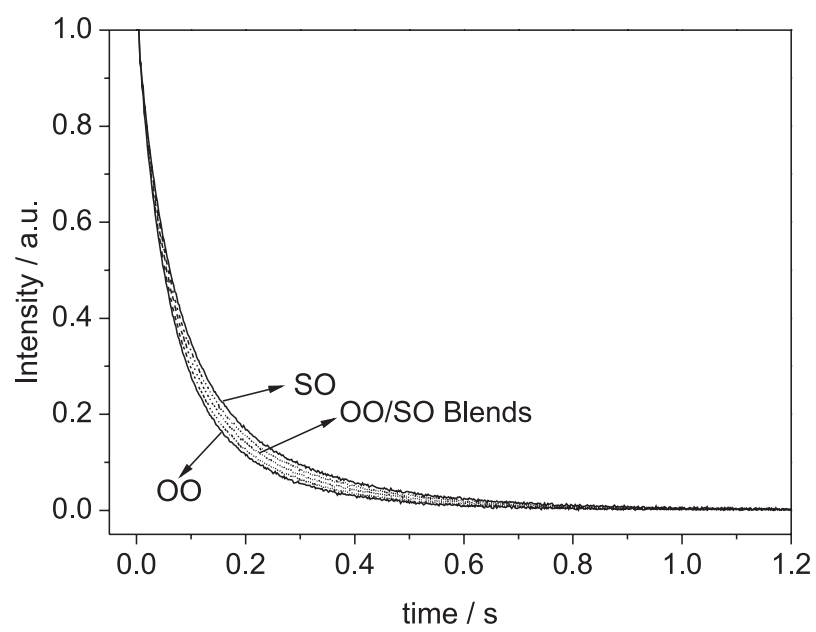

Figure 2. The normalized $\mathrm{T}_{2}$ relaxation curves of $(\mathrm{OO})$ (solid line), $\mathrm{OO} / \mathrm{SO}$ blends comprising 25,50 or $75 \%$ of SO (dashed lines), and SO (solid line). 
Table 1. Fatty acid content percentage of olive and soybean oils as determined by gas chromatography

\begin{tabular}{lccccc}
\hline & Oleic acid & Linoleic acid & Linolenic acid & Palmitic acid & \multicolumn{2}{c}{ Stearic acid } \\
C18:1 & C18:2 & C18:3 & C16:0 & 12.9 & 3.2 \\
\hline Olive oil & 79.2 & 2.7 & 0.6 & 9.6 & 4.2 \\
Soybean oil & 24.8 & 52.3 & 9.6 & 8.1 & 4 \\
\hline
\end{tabular}

with eighteen carbons (C18): $\mathrm{OO}$ was shown to be rich in monounsaturated oleic acid (C18:1) whereas SO was rich in polyunsaturated linoleic (C18:2) and linolenic (C18:3) acids (Table 1 ). The viscosity of fatty acids comprising the same number of carbons decreased with an increase in fatty acid unsaturation. ${ }^{15}$

Inverse Laplace transform (ILT) of the $\mathrm{T}_{2}$ relaxation curves, using regularization parameter $\alpha=1$, of OO, 1:1 OO/SO blend, and SO samples are shown in Figure 3. The relaxation spectra showed two strong peaks for each sample, indicating the presence of two proton components in the fatty acid chains. ${ }^{16} \mathrm{~T}_{2,1}$ (weak peak close to $0.012 \mathrm{~s}$ ) did not show difference among the samples. On the other hand, $\mathrm{T}_{2,2}$ values ranged from 0.044 (OO sample) to $0.056 \mathrm{~s}$ ( $\mathrm{SO}$ sample), while $\mathrm{T}_{2,3}$ values ranged from 0.148 (OO sample) to $0.214 \mathrm{~s}$ (SO sample). Similar $\mathrm{T}_{2}$ values were obtained from an exponential fitting.

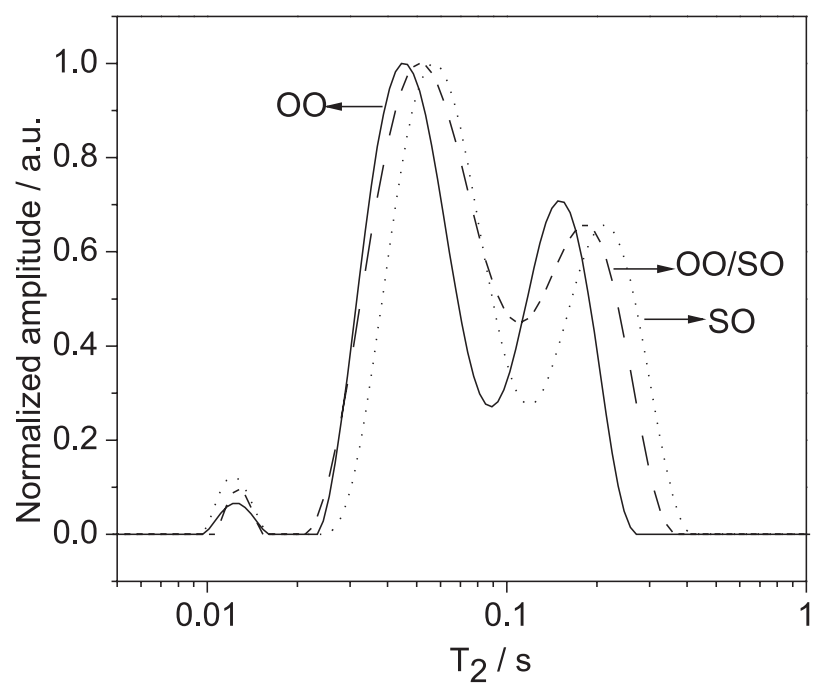

Figure 3. Inverse Laplace transform (ILT) of the CPMG curves of OO (solid line), 1:1 OO/SO blends, and SO (dashed line).

PCA was performed on the maximum normalized $\mathrm{T}_{2}$ relaxation curves (Figure 4). The first principal component (PC1) explained $96.8 \%$ of the data variation and clearly discriminated the samples (OO/SO blends) according to the adulteration level. The second principal component (PC2) explained most of the remaining data variation (3.2\%). The easy separation of the samples based on the adulteration level implies that the application of TD-NMR for distinguishing $\mathrm{OO}$ from $\mathrm{OO} / \mathrm{SO}$ is simple and, as a result, the method may be applied in commercial samples.

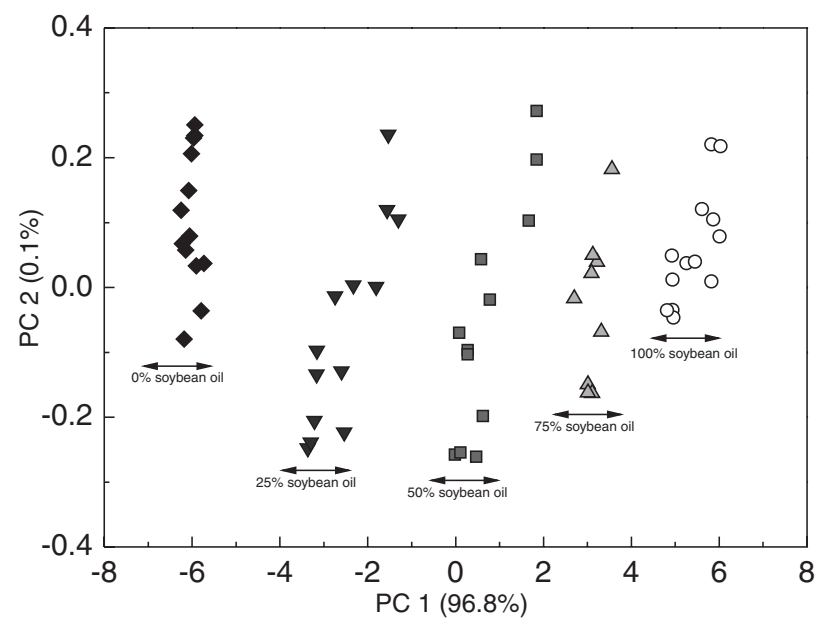

Figure 4. PCA score plot of the full $\mathrm{T}_{2}$ relaxation curves of pure olive oil $(\bullet)$, blends of olive and soybean oils comprising $25(\nabla), 50(\square)$ or $75 \%(\mathbf{\Delta})$ of soybean oil, and pure soybean oil $(\bigcirc)$.

\section{Non-invasive screening of commercial samples}

A procedure used to check oil adulteration relies upon the determination of the ratio between the areas corresponding to the signals at 2.3 and 4.9-5.4 ppm, obtained by ${ }^{1} \mathrm{H} \mathrm{NMR}^{4}$ Figure 5a shows the results obtained for the 37 commercial oil samples. Samples 1-4 were corn, canola, sunflower, and soybean commercial oils. samples 5-7 were commercial blends of olive oil and soybean, canola, sunflower and corn oils. Sample 8 was hazelnut oil, and samples 9-37 are extra virgin olive oils from different countries.

According to the results (Figure 5a), a ratio higher than 0.2 was observed for the samples 1-7, indicating high contents of polyunsaturated fatty acids. This is in agreement with the analyzed samples (soybean, sunflower, corn, canola, etc.). Most of the commercial extra virgin olive oil and hazelnut oil samples showed ratios lower than 0.2, expect for the olive oil samples 14, 20, 29, 30 and 36. This result suggests a possible adulteration with soybean oil or other polyunsaturated oils.

Due to the high cost of this analysis, a new procedure based on the correlation of $T_{2}$ values with the presence of polyunsaturated fatty acids in olive oils was proposed. Although the ILT spectra (Figure 3) showed three $T_{2}$ 
components for all oil samples, the best results were obtained by applying a mono-exponential fitting to the $\mathrm{T}_{2}$ relaxation curves. The obtained results can be observed in Figure 5b. The polyunsaturated oils and blends of olive oil and polyunsaturated oils (samples 1 to 7 ) showed $\mathrm{T}_{2}$ values higher than $0.11 \mathrm{~s}$. Most of the commercial extra virgin olive oil and hazelnut oil samples showed $\mathrm{T}_{2}$ values below $0.11 \mathrm{~s}$, indicating low polyunsaturated fatty acid contents. However, samples 14, 20, 29, 30 and 36 showed $\mathrm{T}_{2}$ values greater than $0.11 \mathrm{~s}$, suggesting adulteration with polyunsaturated oils.
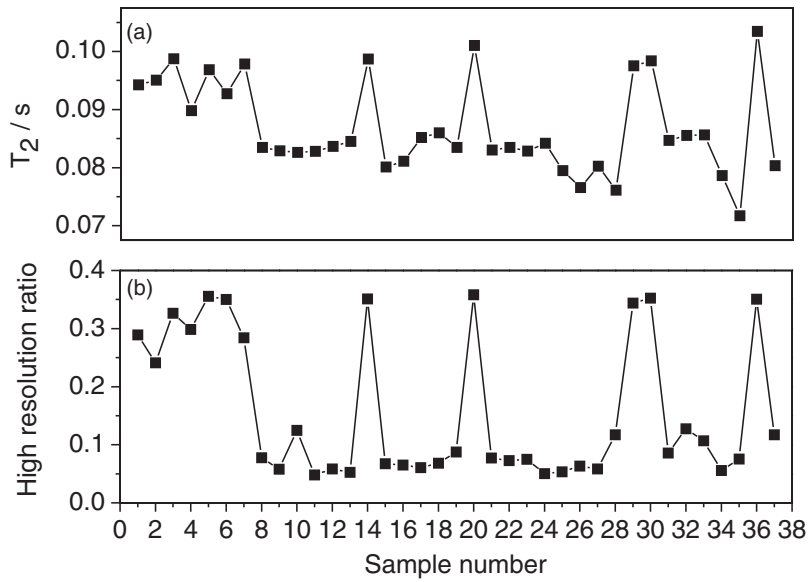

Figure 5. Correlation of commercial oil samples with (a) $T_{2}$ values obtained by mono-exponential fitting of the $\mathrm{T}_{2}$ relaxation curves of 37 commercial oil samples and (b) ratio of the peaks areas at 2.3 (linoleic acid) and 5.5-5.9 ppm (total hydrogens of unsaturated carbons).

Another alternative is the use of chemometric analysis to develop unsupervised and supervised models using the raw $\mathrm{T}_{2}$ relaxation curves. As demonstrated elsewhere in the literature, chemometric analysis of relaxation data has shown better correlations than fitting procedures. ${ }^{16}$ The $37 \mathrm{~T}_{2}$ relaxation curves of the commercial oil samples were first studied using PCA. The first two principal components (PC1 and PC2) explained, together, 97.8\% of the data variation. PCA score plot (Figure 6) showed three clusters: $(i)$ samples 1-7 that, according to the manufacturer's labeling, were polyunsaturated oils (corn, canola, sunflower, soybean or blends of olive oil and soybean, canola, sunflower, and corn oils); (ii) samples 14, 20, 29, 30 and 36 that, based on their labels, were extra virgin olive oil, but showed $\mathrm{T}_{2}$ higher than $0.11 \mathrm{~s}$ (Figure 5); (iii) hazelnut (sample 8) and olive oil samples (9-13, 15-19, 21-28, 31-35 and 37). The hazelnut oil is indicated by a square symbol in Figure 6.

The SIMCA model obtained with the $37 \mathrm{~T}_{2}$ relaxation curves of the commercial oil samples showed a good separation between the polyunsaturated oil and olive oil samples, and interclass distance (ICD) of 3.25. The

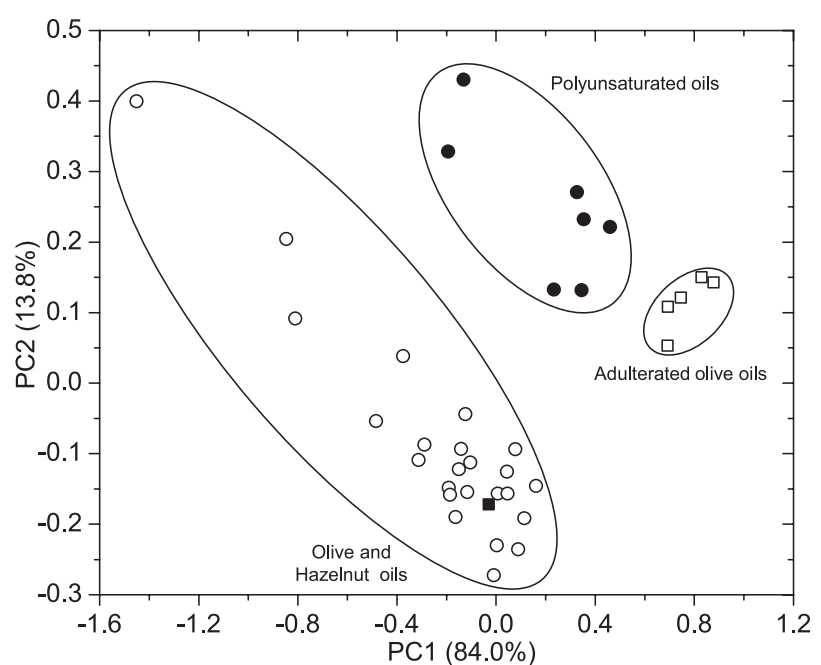

Figure 6. PCA score plot of the full $\mathrm{T}_{2}$ relaxation curves of $(\boldsymbol{O}$ polyunsaturated, $(\bigcirc)$ olive, $(\square)$ hazelnut and $(\square)$ adulterated olive oil samples.

SIMCA classification performance showed that 100 and $83 \%$ of the commercial oil samples were correctly classified as polyunsaturated and olive oil, respectively. Only five commercial olive oil samples were classified as polyunsaturated oil. This agrees with the results shown in Figure 5, where the same olive oil samples (14, 20, 29, 30, and 36) showed ratios higher than 0.2 in ${ }^{1} \mathrm{H}$ NMR and $\mathrm{T}_{2}$ above $0.11 \mathrm{~s}$ in TD-NMR analyses. For the olive oil sample assigned by the number 29, similar result was reported by Instituto Adolfo Lutz (IAL) and Fundação Ezequiel Dias (Funed), as described by the Brazilian Health Surveillance Agency (ANVISA). ${ }^{17}$ No reports were found on the quality and/or authenticity of the other samples.

\section{Conclusions}

These results corroborated the ability of TD-NMR to discriminate olive oil adulterated with high contents of polyunsaturated oil in intact commercial bottles. The only restriction is the oil bottled in metallic containers because of the radiofrequency attenuation. By comparing the performance of univariate and multivariate analyses, the multivariate one is simpler, faster and requires neither a mono- nor a multi-exponential fitting procedure. Overall, TD-NMR has the potential to be used for quality control purposes in different steps of the production chain as well as at any point of the distribution chain, standing out as an attractive procedure for field applications.

\section{Acknowledgments}

The authors would like to acknowledge the Brazilian agencies FAPESP (2011/11160-3, 2012/23169-8, 
2013/03770-1 and 2014/22126-9), CNPq (380777/2014-2) and CAPES (1541262) for their financial support towards this research.

\section{References}

1. Moore, J. C.; Spink, J.; Lipp, M.; J. Food Sci. 2012, 77, 118.

2. Frankél, E. N.; J. Agric. Food Chem. 2010, 58, 5991.

3. Šmejkalová, D.; Piccolo, A.; Food Chem. 2010, 118, 153.

4. Xu, Z.; Morris, R. H.; Bencsik, M.; Newton, M. I.; Sensors 2014, 14, 2028.

5. Aparicio, R.; Aparicio-Ruız, R.; J. Chromatogr. A 2010, 881, 93.

6. Marriott, P. J.; Shellie, R.; Cornwell, C.; J. Chromatogr. A 2001, 936, 1.

7. Parker, T.; Limer, E.; Watson, A. D.; Defernez, M.; Williamson, D.; Kemsley, E. K.; TrAC, Trends Anal. Chem. 2014, 57, 147.

8. Vlachos, N.; Skopelitis, Y.; Psaroudaki, M.; Konstantinidou, V.; Chatzilazarou, A.; Tegou, E.; Anal. Chim. Acta 2006, 573, 459.

9. El-Abassy, R. M.; Donfack, P.; Materny, A.; J. Raman Spectrosc. 2009, 40, 1284.

10. Torrecilla, J. S.; Rojo, E.; Domínguez, J. C.; Rodríguez, F.; J. Agric. Food Chem. 2010, 58, 1679.
11. Maria, M. R.; Colnago, L. A.; Forato, L. A.; Bouchard, D.; J. Agric. Food Chem. 2010, 58, 6562.

12. Perlo, J.; Casanova, F.; Blümich, B.; J. Magn. Reson. 2005, 176, 64.

13. Borgia, G.; Brown, R. J.; Fantazzini, P.; J. Magn. Reson. 2000, 147,273

14. Colnago, L. A.; Andrade, F. D.; Souza, A. A.; Azeredo, R. B. V.; Lima, A. A.; Cerioni, L. M.; Osán, T. M.; Pusiol, D. J.; Chem. Eng. Technol. 2014, 37, 191.

15. Berman, P.; Meiri, N.; Colnago, L. A.; Moraes, T. B.; Linder, C.; Levi, O.; Parmet, Y.; Saunders, M.; Wiesman, Z.; Biotechnol. Biofuels 2015, 8, 12.

16. Santos, P. M.; Côrrea, C. C.; Forato, L. A.; Tullio, R. R.; Cruz, G. M.; Colnago, L. A.; Food Control 2014, 38, 204.

17. http://www.portaldoconsumidor.gov.br/noticia.asp?id=28029, accessed in June 2016.

Submitted: April 19, 2016

Published online: June 21, 2016

FAPESP has sponsored the publication of this article. 